\title{
Uso de microbiota cecal congelada com crioprotetores em pintos infectados experimentalmente com Salmonella enterica sorovar Enteritidis
}

\author{
[Use of frozen cecal microbiota with cryoprotectors in chicks experimentally infected with \\ Salmonella enterica serotype Enteritidis] \\ R.L. Andreatti Filho, E.T. Lima, A.S. Okamoto, H.M. Sampaio \\ Faculdade de Medicina Veterinária e Zootecnia - UNESP \\ Distrito de Rubião Jr, s/n \\ 18618-000 - Botucatu, SP
}

\begin{abstract}
RESUMO
Pintos de corte com um dia de idade foram tratados com microbiota cecal cultivada em condição de aerobiose, nos tempos de congelamento de 90, 200, 290 e 360 dias, e associada aos crioprotetores sacarose, trealose, dimetilsulfóxido (DMSO) e glicerol. Posteriormente as aves foram desafiadas com Salmonella Enteritidis, visando determinar a eficácia dos tratamentos em relação à quantidade de bactérias viáveis da microbiota que foi maior aos 90 dias $\left(10,58 \log _{10} \mathrm{UFC} / \mathrm{ml}\right)$, quando as aves foram tratadas com sacarose, e menor aos 290 dias, quando tratadas com glicerol $\left(7,73 \log _{10} \mathrm{UFC} / \mathrm{ml}\right)$. No tempo zero, todas as aves apresentaram Salmonella $(100 \%)$ quando tratadas com DMSO e glicerol, com colonização cecal de 4,9 e 5,2 $\log _{10} \mathrm{UFC} / \mathrm{g}$ do conteúdo cecal, respectivamente; aos 360 dias nenhuma ave foi infectada, independente do tratamento. A microbiota cecal, independente de tratamento, sempre determinou menor quantidade de $S$. Enteritidis em qualquer um dos parâmetros pesquisados, quando comparada com a das aves não tratadas. $\mathrm{O}$ congelamento em nitrogênio líquido foi eficaz na manutenção da viabilidade da microbiota cecal até 360 dias.
\end{abstract}

Palavras-chave: pinto, microbiota cecal, Salmonella, exclusão competitiva, crioprotetor

\begin{abstract}
One-day-old broiler chicks were treated with cecal microbiota cultivated under aerobiose conditions, frozen during 90, 200, 290 and 360 days and associated with different cryoprotectors such as sucrose, trehalose, DMSO and glycerol. Subsequently, the birds were challenged with Salmonella Enteritidis in order to determine the efficacy of the different treatments in relation to the quantity of viable bacteria, which was higher at 90 days when treated with sucrose $\left(10.58 \log _{10} \mathrm{CFU} / \mathrm{ml}\right)$ and lower at 290 days when treated with glycerol $(7.73$ $\left.\log _{10} \mathrm{CFU} / \mathrm{ml}\right)$. The quantity of infected birds was $100 \%$ in 0 time, when the cecal colonization by $\mathrm{S}$. Enteritidis was 4.9 and $5.2 \log _{10}$ CFU/g of cecal content, respectively treated with DMSO and glycerol. No bird was infected at 360 days, irrespectively of the treatment. In all treatments, the cecal microbiota always determined a lesser quantity of $\mathrm{S}$. Enteritidis for all the studied parameters compared to non-treated birds. Frozen in liquid nitrogen was effective in maintaining the viability of cecal microbiota during the experimental period of 360 days.
\end{abstract}

Keywords: chicken, cecal microbiota, Salmonella, competitive exclusion, cryoprotector

\section{INTRODUÇÃO}

Produtos probióticos e de exclusão competitiva vêm sendo usados na prevenção da infecção de aves por salmonelas. Estes podem ser administrados via oral (Snoeyenbos et al., 1985; Andreatti Filho et al., 1997), por pulverização sobre as aves (Corrier et al., 1994), cama reutilizada (Corrier et al., 1992), via cloaca, in/ex ovo (Corrier et al., 1994; Edens et al., 1997) e na

Recebido em 6 de setembro de 2005

Aceito em 2 de abril de 2007

E-mail: andreatti@fmvz.unesp.br 
forma liofilizada em cápsulas (Corrier et al., 1994). Com a expansão da indústria avícola, a salmonelose adquiriu importância relevante entre as demais doenças aviárias, devido às perdas econômicas e aos problemas de saúde pública (Gast, 1997).

O uso de cultivos de fezes frescas e de conteúdo cecal integral tem se mostrado mais efetivo na prevenção da colonização por Salmonella $\mathrm{sp}$. em relação a produtos que utilizam culturas puras (Stavric et al., 1985). Tanto a cultura aeróbia quanto a anaeróbia de conteúdo cecal têm mostrado resultados protetores (Andreatti Filho et al., 2003). A vida de prateleira, por meio da conservação desses produtos na forma ativa, constitui um importante avanço tecnológico. Dentre os vários métodos de conservação das bactérias (Costa e Ferreira, 1991) destaca-se o congelamento em nitrogênio líquido a $-190^{\circ} \mathrm{C}$, que promove a cristalização das bactérias e evita a sua desidratação (Mazur, 1970; Moore et al., 1998;). Fatores intrínsecos ao congelamento, tais como o choque térmico (Baati et al., 2000), alteração da concentração de soluto intra e extracelular (Ludlan et al., 1989), desidratação (Araújo, 1996) e formação de gelo intra ou extracelular (Jackson, 1997), podem determinar perda de efetividade das bactérias.

Para prevenir ou reduzir os efeitos adversos dos processos de manutenção dos microrganismos, algumas substâncias crioprotetoras, como a sacarose, trealose, dimetilsulfóxido (DMSO), glicerol, entre outras, são adicionadas às amostras bacterianas (Baati et al., 2000). Devido às suas propriedades de preservação contra efeitos danosos à integridade das membranas e paredes de células eucariontes e procariontes, respectivamente, estes podem ser usados nas concentrações de $10 \%$ a $15 \%$, determinando boa criopreservação de Escherichia coli, Klebsiella sp., Serratia marcescens, Lactobacillus sp., Pasteurella multocida, Pseudomonas aeruginosa, entre outras bactérias (Ludlam et al., 1989; Baati et al., 2000). Concentrações de 20\%, ou mais, tanto de DMSO quanto de glicerol, podem causar efeitos danosos às bactérias, não apresentando bons índices de sobrevivência das amostras congeladas (Labruna et al., 1999).

O objetivo deste trabalho foi estudar a capacidade de proteção da microbiota cecal cultivada em condições de aerobiose, associada à sacarose, trealose, DMSO ou glicerol e congelada em nitrogênio líquido, sobre pintos de corte desafiados experimentalmente com Salmonella Enteritidis.

\section{MATERIAL E MÉTODOS}

Utilizou-se amostra de Salmonella enterica sorovar Enteritidis fagotipo 04, mutante resistente ao ácido nalidíxico (Nal) e à rifampicina (Rif), ambos na concentração de $100 \mu \mathrm{g} / \mathrm{ml}$ de meio de cultura (Weinack et al., 1982; Andreatti Filho et al., 1997; 2000).

Os inóculos foram constituídos por culturas de $S$. Enteritidis em $25 \mathrm{ml}$ de caldo infusão de cérebro e coração (BHI), incubados a $40^{\circ} \mathrm{C}$ por 12 horas e diluídos 100 vezes, também em caldo BHI, no momento do uso. Determinou-se o número de unidades formadoras de colônia (UFC) nos inóculos, mediante plaqueamento de $0,1 \mathrm{ml}$ das respectivas diluições decimais em solução salina fosfatada tamponada (PBS) em ágar verde brilhante (BGA) com pH 7,2. Realizou-se a leitura das placas após incubação a $40^{\circ} \mathrm{C}$ por 24 horas.

O desafio foi realizado após 72 horas da administração de cada tratamento da microbiota cecal. As aves receberam $0,5 \mathrm{ml}$ da cultura desafio diluída $\left(3,0 \times 10^{6} \mathrm{UFC} / \mathrm{ml}\right)$, por via intraesofágica e com auxílio de pipeta graduada de $1 \mathrm{ml}$.

Utilizaram-se aves de corte de linhagem comercial, com um dia de idade e não sexadas. Todas alojadas em gaiolas de arame e mantidas sob aquecimento. Ração comercial não medicada e água foram fornecidas ad libitum durante todo o período experimental. A ausência de Salmonella $\mathrm{sp}$. nas aves foi comprovada por meio de metodologia consagrada (Mallinson e Snoeyenbos, 1989).

Cada um dos grupos de aves recebeu, via intraesofágica, os tratamentos com microbiota cecal (MC) cultivada em aerobiose, após congelamento com sacarose, trealose, DMSO ou glicerol, no primeiro dia de vida. A MC para cada grupo experimental foi obtida coletando-se os cecos de 25 aves adultas, livres de patógenos específicos (SPF), sendo cinco aves para cada tratamento. Os cecos, triturados, e seus 
respectivos conteúdos, imersos em caldo tioglicolato sem dextrose (1:10), acrescidos de $100 \mathrm{mMol} / 1$ de sacarose, ou $100 \mathrm{mMol} / 1$ de trealose, ou $10 \%$ de DMSO ou $10 \%$ de glicerol, foram distribuídos em criotubos de $1,8 \mathrm{ml}$ e identificados individualmente. A MC sem $\mathrm{O}$ acréscimo de crioprotetor compôs o grupocontrole do crioprotetor, e aves não tratadas com MC constituíram o grupo-controle da $S$. Enteritidis. Os criotubos foram resfriados à temperatura de $2^{\circ}$ a $8^{\circ} \mathrm{C}$ durante quatro horas. Posteriormente, foram colocados em vapor de nitrogênio, com temperatura variando de $-90^{\circ}$ a $120^{\circ} \mathrm{C}$, durante 20 minutos. Após esse período, foram imersos em nitrogênio líquido $\left(-196^{\circ} \mathrm{C}\right)$ e estocados até o momento de uso.

A determinação do número de UFC da MC foi realizada utilizando-se quatro amostras de cada tratamento, imediatamente antes (tempo zero) e após o congelamento e posterior ao cultivo em aerobiose.

As determinações do inóculo e do número de UFC das MC foram feitas pelo plaqueamento de $0,1 \mathrm{ml}$ das suspensões em caldo BHI e respectivas diluições decimais (PBS), em duplicata, em ágar cérebro coração (BHA). Realizou-se a leitura das placas após incubação a $40^{\circ} \mathrm{C}$ durante 24 horas.

Os inóculos da $\mathrm{MC}$, na quantidade de $0,5 \mathrm{ml} / \mathrm{ave}$, foram administrados via intra-esofágica no primeiro dia de idade das aves. No tempo zero (antes do congelamento) e aos 90, 200, 290 e 360 dias após o congelamento, 24 criotubos, contendo os diferentes crioprotetores, foram descongelados, e a MC cultivada em condição de aerobiose, durante 24 horas a $40^{\circ} \mathrm{C}$. Sessenta aves para cada período foram distribuídas em seis grupos de 10 aves, sendo um deles o grupocontrole de Salmonella, totalizando 300 aves (5x60).

As 96 horas após o desafio com $S$. Enteritidis, todas as aves foram sacrificadas por deslocamento cervical. Os cecos foram removidos assepticamente e transferidos individualmente para bolsas plásticas esterilizadas $\mathrm{O}$ conteúdo cecal foi diluído com PBS (1:10), seguindo-se diluições decimais seriadas em tubos de ensaio contendo PBS e posterior plaqueamento de $0,1 \mathrm{ml}$, em duplicatas de AVB Nal/Rif, cultivadas durante 24 horas à temperatura de $40^{\circ} \mathrm{C}$. Uma vez obtido o número de UFC/grama de conteúdo cecal, este foi convertido em $\log _{10}$ para a interpretação dos resultados.

Para comparação do índice de colonização cecal, da quantidade de aves infectadas e de contagem de UFC da MC com os diferentes crioprotetores, foi utilizado o teste não paramétrico KruskalWallis, seguido do teste Dunn de comparações múltiplas, quando as diferenças estatísticas foram significativas. Os testes foram realizados no nível de 5\% de significância (Zar, 1996).

\section{RESULTADOS}

A quantidade de microbiota cecal (MC) cultivada em aerobiose, adicionada de sacarose, trealose, DMSO ou glicerol e do grupo-controle (sem adição de crioprotetor), antes (tempo zero) e após congelamento nos tempos de 90, 200, 290 e 360 dias em nitrogênio líquido, é apresentada na Tab. 1.

A menor quantidade de UFC da MC, acrescida com sacarose, foi obtida no tempo de 360 dias, e a maior após 90 e 200 dias de congelamento. A quantidade de UFC da MC no tempo zero e aos 290 dias de congelamento foram semelhantes entre si e, também, em relação aos demais resultados. A MC acrescida de trealose, após 360 dias de congelamento, apresentou maior número de UFC do que em 90 dias. O número de UFC no tempo zero e após 200 e 290 dias foram semelhantes entre si e aos de 90 e 360 dias. Não ocorreu diferença significativa nas contagens do inóculo da MC, acrescida de DMSO, glicerol e no controle.

No tempo zero, os tratamentos com crioprotetores não diferiram entre si, quanto ao número de UFC da MC. Após 90 dias de congelamento, a MC acrescida de sacarose apresentou quantidade de UFC semelhante à do grupo-controle. Entretanto, foi mais alta que a obtida com a trealose, DMSO e glicerol. O grupo-controle apresentou quantidade semelhante de UFC quando comparado com o tratado com glicerol e com os grupos que utilizaram DMSO ou trealose. No tempo de 200 dias de congelamento da $\mathrm{MC}$, as maiores contagens da UFC foram as obtidas no tratamento com sacarose e no grupo-controle, seguidos pelo grupo do DMSO. A menor contagem de UFC ocorreu com a MC acrescida de glicerol. Após 290 dias de congelamento da 
MC, a menor quantidade de UFC foi observada no tratamento com glicerol, enquanto a maior quantidade ocorreu no grupo tratado com sacarose ou no grupo-controle. Após 360 dias de congelamento da $\mathrm{MC}$, a menor contagem de UFC foi observada no tratamento com sacarose, e a maior no tratado com glicerol.

Tabela 1. Quantidade de microbiota cecal, cultivada em aerobiose, conservada com diversos crioprotetores, antes e após congelamento (expressas em $\log _{10}$ do número de unidades formadoras de colônia/ml)

\begin{tabular}{|c|c|c|c|c|c|}
\hline \multirow{2}{*}{ Tratamento } & \multicolumn{5}{|c|}{ Tempo de congelamento (dias) } \\
\hline & 0 & 90 & 200 & 290 & 360 \\
\hline \multirow{5}{*}{ Sacarose } & 9,72 & 10,26 & 10,49 & 10,11 & 8,72 \\
\hline & 9,85 & 10,81 & 10,39 & 10,08 & 8,78 \\
\hline & 10,45 & 10,53 & 10,48 & 10,19 & 8,68 \\
\hline & 9,82 & 10,72 & 10,31 & 10,12 & 8,73 \\
\hline & $\overline{\mathrm{x}}=9,96 \mathrm{ABa}$ & $10,58 \mathrm{Bd}$ & $10,42 \mathrm{Bd}$ & $10,13 \mathrm{ABb}$ & $8,73 \mathrm{Aa}$ \\
\hline \multirow{5}{*}{ Trealose } & 9,98 & 9,26 & 9,73 & 9,58 & 9,98 \\
\hline & 10,02 & 9,08 & 9,28 & 9,53 & 9,86 \\
\hline & 9,72 & 9,04 & 9,51 & 9,57 & 9,92 \\
\hline & 7,00 & 9,34 & 9,68 & 9,66 & 9,92 \\
\hline & $\overline{\mathrm{x}}=9,18 \mathrm{ABa}$ & $9,18 \mathrm{Aa}$ & $9,55 \mathrm{ABb}$ & $9,59 \mathrm{ABab}$ & $9,92 \mathrm{Bab}$ \\
\hline \multirow{5}{*}{ DMSO } & 9,73 & 9,56 & 9,76 & 9,59 & 9,51 \\
\hline & 10,08 & 9,72 & 10,00 & 9,85 & 9,71 \\
\hline & 9,32 & 9,70 & 9,95 & 9,58 & 9,62 \\
\hline & 9,34 & 9,87 & 9,78 & 9,71 & 9,61 \\
\hline & $\overline{\mathrm{x}}=9,62 \mathrm{Aa}$ & $9,71 \mathrm{Aab}$ & $9,87 \mathrm{Ac}$ & $9,68 \mathrm{Aab}$ & $9,61 \mathrm{Aab}$ \\
\hline \multirow{5}{*}{ Glicerol } & 11,48 & 10,20 & 9,18 & 7,71 & 9,48 \\
\hline & 11,22 & 10,28 & 9,34 & 7,91 & 9,85 \\
\hline & 6,48 & 9,95 & 9,26 & 7,54 & 10,77 \\
\hline & 0,00 & 9,18 & 9,26 & 7,76 & 10,49 \\
\hline & $\bar{x}=9,73 \mathrm{Aa}$ & $9,90 \mathrm{Abc}$ & $9,26 \mathrm{Aa}$ & $7,73 \mathrm{Aa}$ & $10,15 \mathrm{Aab}$ \\
\hline \multirow{5}{*}{ Controle $^{1}$} & 10,23 & 10,28 & 10,31 & 11,01 & 10,10 \\
\hline & 10,18 & 10,50 & 10,34 & 10,20 & 10,11 \\
\hline & 10,23 & 10,29 & 10,17 & 10,34 & 10,06 \\
\hline & 10,89 & 10,36 & 10,28 & 10,77 & 10,08 \\
\hline & $\overline{\mathrm{x}}=10,38 \mathrm{Aa}$ & 10,36 Acd & $10,28 \mathrm{Ad}$ & $10,58 \mathrm{Ab}$ & $10,09 \mathrm{Ab}$ \\
\hline
\end{tabular}

Valores seguidos por letras maiúsculas distintas na linha dentro do tratamento, ou letras minúsculas distintas na coluna dentro do período diferem entre si $(\mathrm{P}<0,05)$.

${ }^{1}$ Microbiota cecal sem adição de crioprotetor.

A quantidade de aves infectadas por $S$. Enteritidis, tratadas com MC, antes e após congelamento em nitrogênio líquido, adicionada ou não de crioprotetores, é apresentada na Tab. 2.

O tratamento da $\mathrm{MC}$ com sacarose não determinou diferença significativa na quantidade de aves infectadas por $S$. Enteritidis nos cinco períodos de congelamento testados. A MC acrescida de trealose não resultou em aves infectadas por $S$. Enteritidis, após 360 dias de congelamento, enquanto a maior quantidade infectada ( $50 \%$ das aves) foi observada no tempo zero. Os tratamentos da MC com DMSO e glicerol, além do grupo sem crioprotetor, determinaram a maior quantidade de aves infectadas por $S$. Enteritidis no tempo zero, significativamente diferente dos demais períodos. No grupo somente desafiado com $S$. Enteritidis, todas as aves apresentaram-se infectadas em todos os tempos testados. 
Tabela 2. Quantidade de aves infectadas por Salmonella Enteritidis, tratadas com microbiota cecal cultivada em aerobiose, conservada com diversos crioprotetores e congelada em nitrogênio líquido (número de aves infectadas/número de aves tratadas; percentual)

\begin{tabular}{lccccc}
\hline \multirow{2}{*}{ Tratamento } & \multicolumn{5}{c}{ Período de congelamento (dias) } \\
\cline { 2 - 5 } & 0 & 90 & 200 & 290 & 360 \\
\hline Sacarose & $0 / 10(0) \mathrm{Aa}$ & $0 / 10(0) \mathrm{Aa}$ & $0 / 10(0) \mathrm{Aa}$ & $3 / 10(30) \mathrm{Aa}$ & $0 / 10(0) \mathrm{Aa}$ \\
Trealose & $5 / 10(50) \mathrm{Bb}$ & $2 / 10(20) \mathrm{ABa}$ & $2 / 10(20) \mathrm{ABa}$ & $2 / 10(20) \mathrm{ABa}$ & $0 / 10(0) \mathrm{Aa}$ \\
DMSO & $10 / 10(100) \mathrm{Bc}$ & $0 / 10(0) \mathrm{Aa}$ & $3 / 10(30) \mathrm{Aa}$ & $0 / 10(0) \mathrm{Aa}$ & $0 / 10(0) \mathrm{Aa}$ \\
Glicerol $_{\text {Controle crioprotetor }}{ }^{1}$ & $10 / 10(100) \mathrm{Bc}$ & $4 / 10(40) \mathrm{Aa}$ & $0 / 10(0) \mathrm{Aa}$ & $1 / 10(10) \mathrm{Aa}$ & $0 / 10(0) \mathrm{Aa}$ \\
Controle Salmonella $^{2}$ & $6 / 10(60) \mathrm{Bbc}$ & $0 / 10(0) \mathrm{Aa}$ & $1 / 10(10) \mathrm{Aa}$ & $0 / 10(0) \mathrm{Aa}$ & $0 / 10(0) \mathrm{Aa}$ \\
\hline
\end{tabular}

Valores seguidos por letras maiúsculas distintas na linha dentro do tratamento, ou letras minúsculas distintas na coluna dentro do período diferem entre si $(\mathrm{P}<0,05)$.

${ }^{1}$ Aves que receberam microbiota cecal, sem crioprotetor, e desafiadas com $S$. Enteritidis.

${ }^{2}$ Aves somente desafiadas com $S$. Enteritidis.

No tempo zero, o grupo que recebeu MC com sacarose não apresentou aves infectadas por $S$. Enteritidis, significativamente diferente dos demais tratamentos. Nos demais tempos de congelamento, somente o grupo desafiado com $S$. Enteritidis e não tratado apresentou $100 \%$ das aves infectadas, diferindo de todos os demais tratamentos.
A colonização cecal de $S$. Enteritidis em aves tratadas com MC, antes e após congelamento em nitrogênio líquido, adicionada ou não de crioprotetores, é apresentada na Tab. 3.

Tabela 3. Colonização cecal de Salmonella Enteritidis em aves tratadas com microbiota cecal, conservada com diversos crioprotetores e cultivada em aerobiose (média de 10 aves, expressa em $\log _{10}$ do número de unidades formadoras de colônia/grama de conteúdo cecal)

\begin{tabular}{lccccc}
\hline \multirow{2}{*}{ Tratamento } & \multicolumn{4}{c}{ Período de congelamento (dias) } \\
\cline { 2 - 5 } & 0 & 90 & 200 & 290 & 360 \\
\hline Sacarose & $0,0 \mathrm{Aa}$ & $0,0 \mathrm{Aa}$ & $0,0 \mathrm{Aa}$ & $1,3 \mathrm{Aa}$ & $0,0 \mathrm{Aa}$ \\
Trealose & $1,9 \mathrm{Aab}$ & $0,8 \mathrm{Aa}$ & $0,5 \mathrm{Aa}$ & $0,4 \mathrm{Aa}$ & $0,0 \mathrm{Aa}$ \\
DMSO & $4.9 \mathrm{Bcd}$ & $0,0 \mathrm{Aa}$ & $1,3 \mathrm{Aa}$ & $0,0 \mathrm{Aa}$ & $0,0 \mathrm{Aa}$ \\
Glicerol & $5,2 \mathrm{Bd}$ & $1,4 \mathrm{Aa}$ & $0,0 \mathrm{Aa}$ & $0,3 \mathrm{Aa}$ & $0,0 \mathrm{Aa}$ \\
Controle crioprotetor & $2,2 \mathrm{Babc}$ & $0,0 \mathrm{Aa}$ & $0,3 \mathrm{Aa}$ & $0,0 \mathrm{Aa}$ & $0,0 \mathrm{Aa}$ \\
Controle Salmonella $^{2}$ & $4,9 \mathrm{Abcd}$ & $4,5 \mathrm{Ab}$ & $5,5 \mathrm{Ab}$ & $3,8 \mathrm{Ab}$ & $5,4 \mathrm{Ab}$ \\
\hline
\end{tabular}

Valores seguidos por letras maiúsculas distintas na linha dentro do tratamento, ou letras minúsculas distintas na coluna dentro do período diferem entre si $(\mathrm{P}<0,05)$.

${ }^{1}$ Aves que receberam microbiota cecal sem crioprotetor e desafiadas com $S$. Enteritidis.

${ }^{2}$ Aves somente desafiadas com $S$. Enteritidis.

Não ocorreram diferenças significativas na colonização cecal de aves tratadas com $\mathrm{MC}$ acrescida de sacarose ou trealose e no controle de $S$. Enteritidis nos cinco períodos estudados. A MC, acrescida de DMSO, glicerol ou sem crioprotetor, resultou em maior colonização cecal por $S$. Enteritidis no tempo zero em relação aos demais períodos experimentais.

No tempo zero, não se observou colonização cecal por $S$. Enteritidis nas aves tratadas com MC acrescida de sacarose, tornando-as significativamente distintas das aves dos grupos que receberam MC acrescida de DMSO e glicerol ou mesmo do grupo desafiado somente com $S$. Enteritidis. Todos os grupos de aves que receberam MC com ou sem crioprotetor, nos tempos de congelamento de 90, 200, 290 e 360 dias, apresentaram colonização cecal significativamente menor que a do grupo desafiado somente com $S$. Enteritidis.

\section{DISCUSSÃO}

Produtos probióticos e de exclusão competitiva podem ser administrados via oral (Snoeyenbos et al., 1985; Andreatti Filho et al., 1997), por pulverização sobre as aves (Corrier et al., 1994), cama reutilizada (Corrier et al., 1992), via 
cloaca, in/ex ovo (Corrier et al., 1994; Edens et al., 1997) e na forma liofilizada em cápsulas (Corrier et al., 1994). A via intra-esofágica utilizada permitiu maior uniformidade da MC, minimizando as eventuais variações nos resultados. Bactérias probióticas podem ser obtidas a partir de fezes (Snoeyenbos et al., 1985), conteúdo cecal (Nurmi e Rantala, 1973; Corrier et al., 1991, 1992; Andreatti Filho et al., 1997, 2000), inglúvio (Nurmi e Rantala, 1973) e cama utilizada (Corrier et al., 1992). O conteúdo cecal apresenta-se mais efetivo na prevenção à colonização por Salmonella sp. (Nurmi e Rantala, 1973; Ziprin et al., 1993).

Não se observou mortalidade nas aves infectadas por $S$. Enteritidis entre os grupos tratados e os grupos-controles, semelhante aos observados por Silva (1992) e Navarro (1995), em que as salmonelas paratifóides determinaram baixa ou nenhuma mortalidade, salvo quando as aves foram imunocomprometidas.

Os compostos destinados à elaboração de probióticos ou produtos de exclusão competitiva têm sido mantidos principalmente por liofilização ou congelamento (Corrier et al., 1991; Andreatti Filho e Crocci, 2002). Entretanto, ainda não são suficientes as informações sobre a estabilidade desse tipo de material sob tais métodos de conservação, ao longo de vários meses, especialmente quando se comparam os resultados obtidos com fezes ou conteúdo intestinal frescos.

O efeito benéfico esperado dos crioprotetores utilizados não foi significativo. Os resultados obtidos nas contagens da MC (Tab. 1), nos diferentes tempos e tratamentos, não comprovaram se a ausência de tais substâncias determinaria resultados mais baixos, visto que o grupo-controle, em vários momentos, foi tão efetivo quanto os resultados obtidos com a adição dos crioprotetores.

A MC conservada, com ou sem crioprotetores, manteve boa efetividade mesmo até 360 dias de congelamento, ao reduzir o número de aves infectadas (Tab. 2) e a colonização cecal (Tab. 3) por $S$. Enteritidis.

Observou-se que o tratamento com o cultivo aeróbio permitiu redução do número de aves infectadas por $S$. Enteritidis nos diversos períodos experimentais, resultados semelhantes aos de Oliveira et al. (2000), Almeida e Berchieri (2001) e Andreatti Filho et al. (2003) que, ao tratarem pintos de um dia com culturas de microbiota intestinal cultivada em aerobiose, obtiveram redução da colonização cecal por Salmonella sp. Apesar de a maioria das pesquisas com probióticos ou produtos de exclusão competitiva enfocar o cultivo anaeróbio, devem ser consideradas as diversas variáveis existentes nos protocolos experimentais empregados, como tempo de cultivo, número de passagens nos meios de cultura, espécies de bactérias que os compõem, via de administração e os sorotipos de Salmonella desafio.

\section{CONCLUSÕES}

O congelamento em nitrogênio líquido mostrouse eficaz na manutenção da viabilidade da microbiota cecal durante o período experimental de 360 dias. O efeito benéfico esperado dos crioprotetores, sacarose, trealose, DMSO ou glicerol não foi significativo. Nas condições experimentais e com os protocolos seguidos, o cultivo aeróbio da microbiota cecal permitiu a redução do número de aves infectadas e da colonização cecal por $S$. Enteritidis.

\section{REFERÊNCIAS BIBLIOGRÁFICAS}

ALMEIDA, W.A.F.; BERCHIERI JR., A. Controle de Salmonella enterica sorovar Enteritidis em aves recémnascida pelo uso de exclusão competitiva. Rev. Bras. Cien. Avic, v.3, supl.1, p.93, 2001.

ANDREATTI FILHO, R.L.; CROCCI, A.J. Efeito protetor da microbiota cecal congelada e liofilizada sobre a infecção experimental de frangos de corte por Salmonella enterica sorovar Enteritidis. Arq. Bras. Med. Vet. Zootec., v.54, p.457-461, 2002.

ANDREATTI FILHO, R.L.; SAMPAIO, H.M.; BARROS, M.R. et al. Use of cecal microflora cultured under aerobic or anaerobic conditions in the control of experimental infection of chicks with Salmonella Enteritidis. Vet. Microbiol., v.92, p.237-244, 2003.

ANDREATTI FILHO, R.L.; SILVA, E.N.; CURI, P.R. Ácidos orgânicos e microbiota cecal anaeróbia no controle da infecção experimental de frangos por Salmonella Typhimurium e Salmonella Enteritidis. Arq. Bras. Med. Vet. Zootec., v.49, p.661-672, 1997.

ANDREATTI FILHO, R.L.; SILVA, E.N.; RIBEIRO, A.R. et al. Use of anaerobic cecal microflora, lactose and acetic acid for the protection of broiler chicks against experimental infection with Salmonella Typhimurium and 
Salmonella Enteritidis. Braz. J. Microbiol., v.31, p.10712, 2000.

ARAÚJO, P.S. The role of trehalose in cell stress. Braz. J. Med. Biol. Res., v.29, p.873-875, 1996.

BAATI, L; FABRE-GEA, C.; AURIOL, D. et al. Study of the cryotolerance of Lactobacillus acidophilus: effect of culture and freezing on the viability and cellular protein levels. Int. J. Food Microbiol., v.59, p.241-247, 2000.

CORRIER, D.E.; HARGIS, B.M.; HINTON JR. A. et al. Effect of anaerobic cecal microflora and dietary lactose on colonization resistence of layer chickens to invasive Salmonella enteritides. Avian Dis., v.35, p.337-343, 1991.

CORRIER, D.E.; HOLLISTER, A.G.; NISBET, D.J. et al. Competitive exclusion of Salmonella enteritides in leghorn chicks: comparision of treatment by crop gavage, drinking water, spray of lyophilized alginate beads. Avian Dis., v.38, p.297-303, 1994.

CORRIER, D.E.; SNODGRASS, J.D.; HINTON JR., A. et al. Effect of anaerobic cecal microflora and dietary lactose on Salmonella enteritides. Poult. Sci., v.71, p.2022-2026, 1992.

COSTA, C.P.; FERREIRA, M.C. Preservação de microrganismos: Revisão. Rev. Microbiol., v.22, p.263266, 1991

EDENS, F.W.; PARKHURST, C.R.; CASAS, I.A. et al. Principles of ex ovo competitive exclusion and in ovo administration of Lactobacillus reuteri. Poul. Sci., v.76, p.179-196, 1997.

GAST, R.K. Paratyphoid Infections, In: CALNEK B. W. (Eds.). Disease of poultry. 10.ed. Ames: Iowa State University, 1997. p.97-121.

JACKSON, T.H.; UNGAN, A.; CRITSER, J.K. et al. Novel microwave technology for cryopreservation of biomaterials by suppression of apparent ice formation. Cryobiology, v.34, p.363-372, 1997.

LABRUNA, M.B.; RESENDE, J.S.; MARTINS, N.R.S. et al. Criopreservação de uma amostra de espiroqueta em nitrogênio líquido. Arq. Bras. Med. Vet. Zootec., v.51, p.551-553, 1999.

LUDLAM, H.A.; NWACHUKWU, B.; NOBLE, W.C. et al. The preservation of micro-organisms in biological specimens stored at $-70^{\circ} \mathrm{C}$. J. Appl. Bacteriol., v.67, 417 $423,1989$.

MALLINSON, E.T.; SNOEYENBOS, G.H Salmonellosis. In: PURCHASE, H.G.; ARP, H.L.;
DOMERMUTH, H.C. et al. (Eds.). A laboratory manual for the isolation and identification of avian pathogens. 3.ed. American Association of Avian Pathologists; Pennsylvania, 1989. p.3-11.

MAZUR, P. Cryobiology: The freezing of biological systems. Science, v.168, p.939-949, 1970.

MOORE, M.K.; SHADDUCK, D.J.; GOLDBERG, D.R. et al. A cryopreservation method for Pasteurella multocida from wetland samples. J. Wildl. Dis., v.34, p.182-185, 1998.

NAVARRO, M.P. Infecção por Salmonella enteritidis em reprodutoras pesadas na América Latina. In: CONFERÊNCIA APINCO DE CIÊNCIA E TECNOLOGIA AVÍCOLAS, 1995, Curitiba. Anais... Curitiba, 1995. p.7-16.

NURMI, E.; RANTALA, M. New aspects of Salmonella infection in broiler production. Nature, v.241, p.210-211, 1973.

OLIVEIRA, E.; BERCHIERI, Jr., A.; BARROW, P.A. Prevention of Salmonella infection by contact using intestinal flora of adult birds and/or a mixture of organic acids. Braz. J. Microbiol., v.31, p.116-120, 2000.

SILVA, E.N. Salmoneloses em galinhas. In: CURSO DE SANIDADE AVÍCOLA. Campinas: Fundação Apinco de Ciência e Tecnologia Avícolas, 1992.

SNOEYENBOS, G.H.; WEINACK, O.M.; SOERJADI, A.S. et al. Large-scale trial to study competitive exclusion of Salmonella in chickens. Avian Dis., v.29, p.1004-1011, 1985.

STAVRIC, S.; GLEESON, T.M.; BLANCHFIELD, B. et al. Competitive exclusion of Salmonella from new hatched chicks by misture of pure bacterial cultures isolated from fecal and cecal contents of adult birds. $J$. Food Protect., v.48, p.778-782, 1985.

WEINACK, O.M.; SNOEYENBOS, G.H.; SMYSER, C.F. et al. Reciprocal competitive exclusion of Salmonella and Escherichia coli by native intestinal microflora of the chicken and turkey. Avian Dis., v.26, p.585-595, 1982.

ZAR, J.H. Biostatistical analisis. 2.ed. Englewood Cliffs: Prentice-Hall, 1996. 718p.

ZIPRIN, R.L.; CORRIER, D.E.; DeLOACH, J.R. Control of established Salmonella typhimurium intestinal colonization with in vivo passaged anaerobes. Avian Dis., v.37, p.183-188, 1993. 